\title{
New approaches in teaching spectroscopy technique and application classes: history, experiments and frontier lectures
}

\section{Qing Yang, Minghua Zhuge, Bo Yuan}

Qing Yang, Minghua Zhuge, Bo Yuan, "New approaches in teaching spectroscopy technique and application classes: history, experiments and frontier lectures," Proc. SPIE 10452, 14th Conference on Education and Training in Optics and Photonics: ETOP 2017, 1045236 (16 August 2017); doi: $10.1117 / 12.2270714$

SPIE Event: 14th Conference on Education and Training in Optics and Photonics, ETOP 2017, 2017, Hangzhou, China 


\title{
New Approaches in Teaching Spectroscopy Technique and Application
}

\section{Classes: History, Experiments and Frontier Lectures}

\author{
Qing Yang *`, Minghua Zhuge ${ }^{\dagger}$, Bo Yuan ${ }^{+}$ \\ 'State Key Laboratory of Modern Optical Instrumentation, College of Optical Science and \\ Engineering, Zhejiang University, Hangzhou 310027, China \\ "E-mail: qingyang@zju.edu.cn \\ Address: Room 418, Building 3, Zheda Road NO.38, Hangzhou, Zhejiang, China
}

\begin{abstract}
Spectroscopy has a long history. The theory is difficult for students to understand. In order to improve the traditional teaching to a way of interesting experience, history story, class experiments and frontier lectures are introduced simultaneously. In the class, we will not only show students a lot of phenomena about spectroscopy in our daily life, but also show them history stories of spectroscopy scientists, which will make them feel close to spectroscopy. Meanwhile, students are suggested to read cutting-edge research papers to obtain the newest information and knowledge about spectroscopy. Typically and importantly, students are required to do experiments in lab and in class. Through this practice, they will understand the theory much more deeply, especially they will know how to solve the problems in research.
\end{abstract}

\section{Introduction}

Spectroscopic investigations give us the most knowledge of the structure of atoms and molecules. Thus spectroscopy helps people a lot to understand the atomic and molecular physics. Information on atomic and molecular structure and how they interplay with surroundings could be studied in various ways from the absorption or emission spectra generated when electromagnetic radiation interacts with matter. This kind of interaction could provide different spectra.

Spectroscopy is a general method which could tell us the basic information of the objects such as electronic levels, molecular vibration states, molecular rotation states, particle structure and symmetry, transition probability and so on. It is the base of physics, chemistry and astronomy. Furthermore, spectroscopy can be utilized in environments monitoring, industrial inspection, clinical medicine, earth view and so on.

The theory of spectroscopy is both related to classic physics and quantum mechanics. It is multidisciplinary in theory and practice. Therefore, as understanding the nature of spectroscopy is difficult for undergraduates, when teaching this course, we truly face some problems.

For better understanding and application of the optical spectroscopy, we introduce history story, frontiers lectures and experiments. We also lead them to play games in class which could help them understand the motions of the atom and molecule better.

\section{History and papers}

Spectroscopy has a long history and lots of stories. Collecting and selecting some milestones from huge historical materials, such as the prismatic decomposition experiment done by Newton in

14th Conference on Education and Training in Optics and Photonics: ETOP 2017, edited by Xu Liu,

Xi-Cheng Zhang, Proc. of SPIE Vol. 10452, 1045236 • () 2017 ICO, IEEE, OSA, SPIE

CCC code: $0277-786 \mathrm{X} / 17 / \$ 18 \cdot$ doi: $10.1117 / 12.2270714$ 
1666 and the first spectroscopy invented by Kirchhoff in 1859, and later, how the scientists discovered Zeemann and Stark splittings by external magnetic or electric fields, will help students deeply understand the technique of spectra.

We also invite doctor candidates who do research in related fields to give lectures. They bring undergraduates the up-to-date information of spectroscopy like the application of CARS (Coherent Anti-starks Raman Scattering) and SRS (Stimulated Raman Scattering) in biomedical sciences and the development of optoacoustic spectroscopy. Undergraduates are asked to read related papers and give presentations in class as well.

\section{Course experiments}

\section{Diode laser based absorption spectroscopy}

In order to help students understand the Doppler limited absorption spectroscopy, we set up the experiments system of diode laser absorption spectroscopy in studying the Rubidium D1 or D2 transitions, which has a well-known energy structure ${ }^{1}$.

In this paper we present experiments with Rubidium, in particular the $794.98 \mathrm{~nm}$ transition. And the optics can easily be rearranged to carry out saturated absorption spectroscopy. The experimental set up is also versatile in the sense that to change the frequency we merely set the appropriate frequency and amplitude of the AC signal from the signal generator which is guided into the current controller connected with the diode laser.

Because the alkali metals, including rubidium, have the same outer electronic configuration as hydrogen, they have relatively simple hydrogen-like energy level structure. As a result of the hyperfine interaction between the nuclear and the valence-electron magnetic moments, the atomic ground state splits into two hyperfine sub-levels characterized by a total angular momentum quantum number $F$. Since the energy of the $P_{1 / 2}$ state is less than the energy of the $P_{3 / 2}$ state, the $D_{1}$ wavelength is always greater than the $\mathrm{D}_{2}$ wavelength. Figure 1 shows the few lowest energy levels of ${ }^{85} \mathrm{Rb}$ and ${ }^{87} \mathrm{Rb}$. 


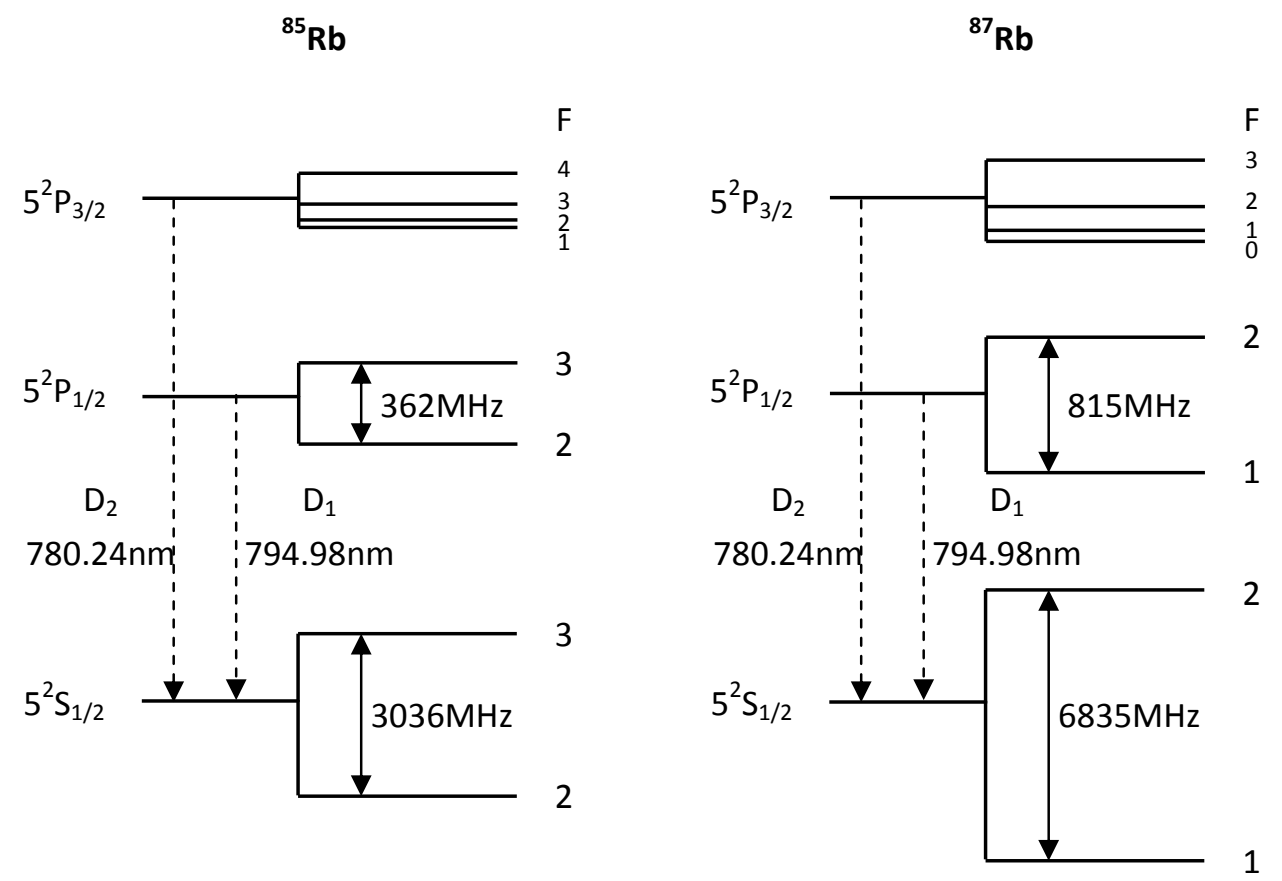

Fig. 1 The few lowest energy levels of ${ }^{85} \mathrm{Rb}$ and ${ }^{87} \mathrm{Rb}$. The $\mathrm{D}_{1}$ line corresponds to a ${ }^{2} \mathrm{P}_{1 / 2}-{ }^{2} \mathrm{~S}_{1 / 2}$ transition and that the $\mathrm{D}_{2}$ line corresponds to $\mathrm{a}^{2} \mathrm{P}_{3 / 2}-{ }^{2} \mathrm{~S}_{1 / 2}$ transition.

Figure 2 shows a schematic layout of the experimental set-up used for laser absorption and laser-induced fluorescence spectroscopy. The diode laser is supplied with current form a low noise diode laser driver. A particular wavelength of the diode laser is obtained by tuning and stabilizing the temperature of the laser capsule using a temperature controller. By using a saw tooth current waveform with a high repetition rate on the laser drive current, the wavelength of the laser is repetitively scanned and an absorption spectrum can be recorded in real time on the oscilloscope screen. A beam splitter directs some of the laser light to allow the relative wavelength scale to be calibrated form the etalon free spectral range. The absorption and calibration spectra are recorded by a digital memory oscilloscope prior to data analysis. 


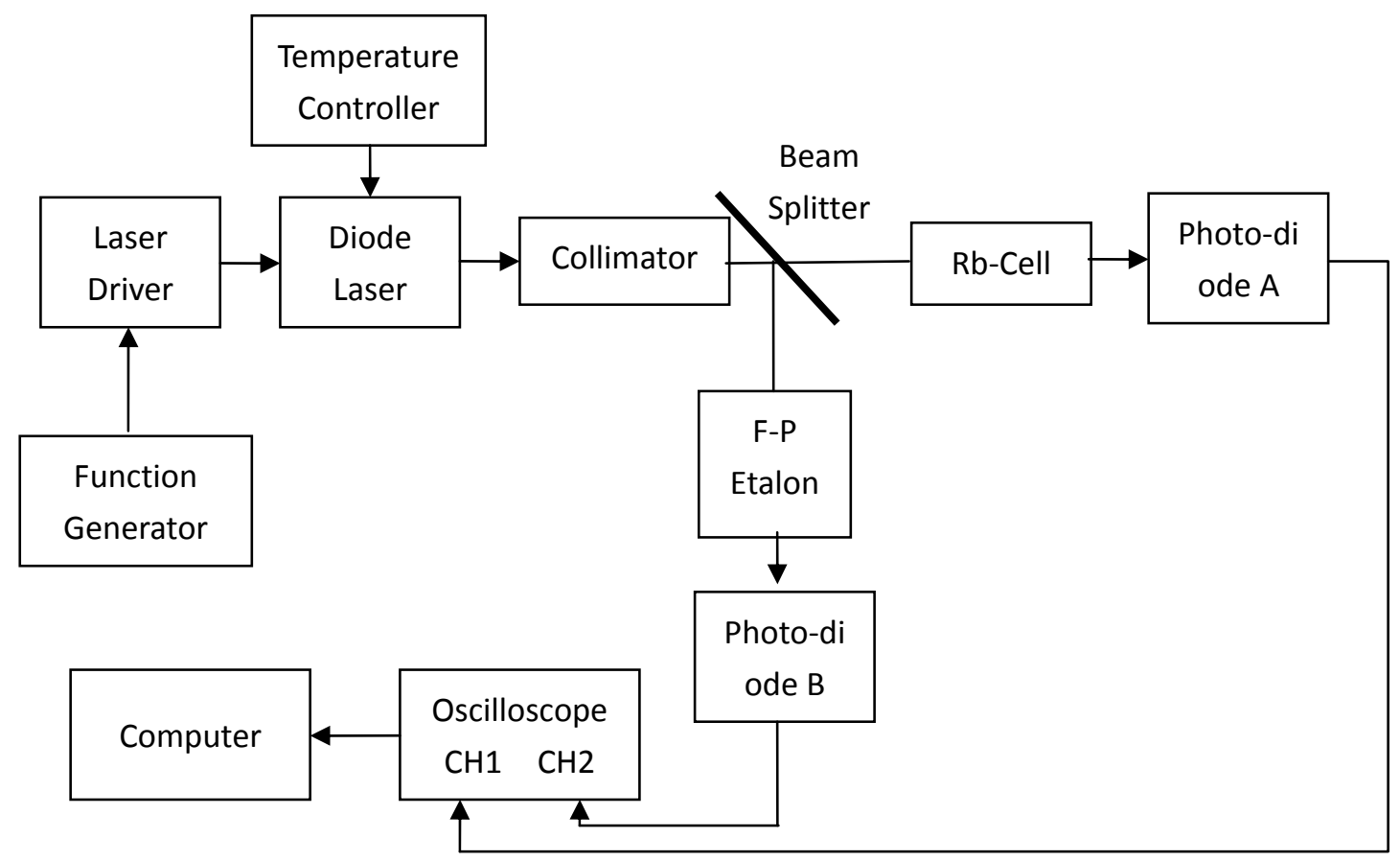

Fig.2 Schematic diagram of the diode laser spectrometer configured for Doppler limited absorption spectroscopy.

Two different rubidium cells are used in our experimental set up, and they contain ${ }^{87} \mathrm{Rb}$ and ${ }^{85} \mathrm{Rb} /{ }^{87} \mathrm{Rb}$ respectively. Through carefully building up the experimental system and controlling the current and temperature of laser diode, undergraduates obtain absorption spectra of both cells. The former has four peaks in $794.98 \mathrm{~nm}$ transfer and the latter has six peaks owing to some energy levels of two isotopes are too close to each other.

Figure 3 shows an absorption spectrum of ${ }^{87} \mathrm{Rb}$ and the corresponding throughout of the solid Fabry-Perot etalon. From this figure alone a student should be able to determine the atom density in the cell, linewidths, and the hyperfine splitting of the ground state.

In this experiment with simple portable and cheap spectrometer, students can understand how diode laser absorption can be used to measure simple parameters in the Rubidium D1 transition $\left(5^{2} \mathrm{P}_{3 / 2}-5^{2} \mathrm{~S}_{1 / 2}\right)$ at $794.98 \mathrm{~nm}$ and then enhance the understanding of Doppler broadening in low pressure gases. 


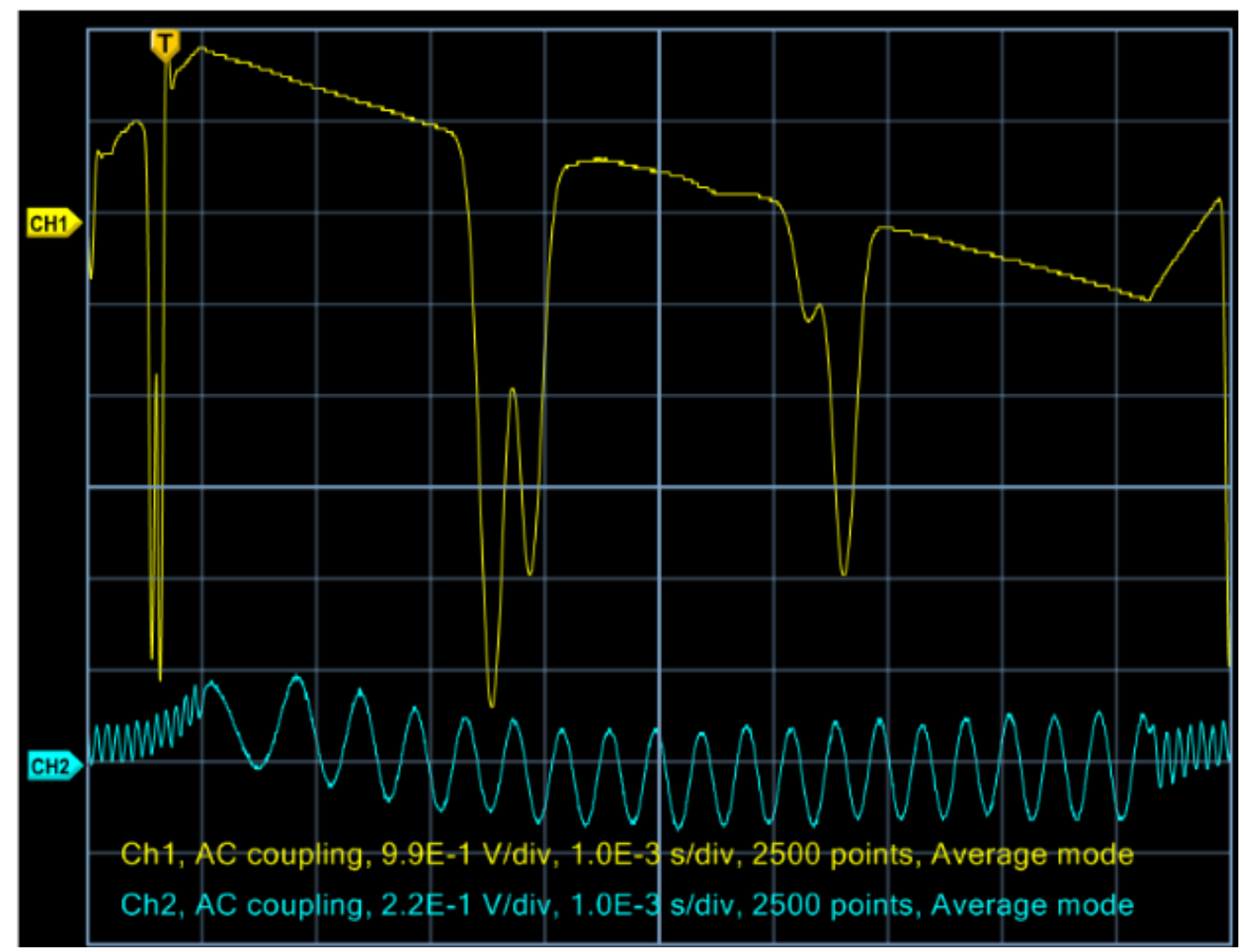

Fig. 3 Doppler limited absoprtion spectra of ${ }^{87} \mathrm{Rb}$ and resonance spectra of a solid Fabry-Perot etalon, which is used for frequency calibration.

\section{Saturated Absorption Spectroscopy}

Saturated absorption experiments were cited in the 1981 Nobel Prize in physics and related techniques have been used in laser cooling and trapping experiments cited in the 1997 Nobel Prize as well as Bose-Einstein condensation experiments cited in the 2001 Nobel Prize. Therefore, saturated absorption spectroscopy is a useful spectrum technology.

According to the aforementioned experiment, we make use of a tunable diode laser to carry out spectroscopic studies of the rubidium atom and measure the Doppler-broadened absorption profiles of the D1 transitions at $794.98 \mathrm{~nm}$. Then we exploit the technique of saturated absorption spectroscopy to improve the resolution beyond the Doppler limit and measure the nuclear hyperfine splitting. In order to reach this goal, we add the necessary optical devices to complete the experiment set up of saturated absorption spectroscopic of the Rubidium atom. As the same to the previous, a Fabry-Perot optical resonator is used to calibrate the frequency scale for the measurements ${ }^{2}$.

The layout of the saturated absorption spectrometer is shown in Figure 4. Collimated LD laser 1 splits by the beam splitter into 2 and 3 . As the pump beam, laser 2 enters the cell to make the Rubidium atoms saturated, and then some of beam 2 will be reflected by the wedge flap to become the weak detective beam 4 . The beam 4 enters the cell again in the opposite direction to detect the saturated spectrum of Rubidium atoms. Through the cell, the beam 4 then is reflected into 
photo-diode A. Beam 3 enters F-P etalon and is detected by photo-diode B. Figure 5 shows a saturated absorption spectrum of ${ }^{85} \mathrm{Rb} /{ }^{87} \mathrm{Rb}$ and the corresponding spectrum of the solid Fabry-Perot etalon.

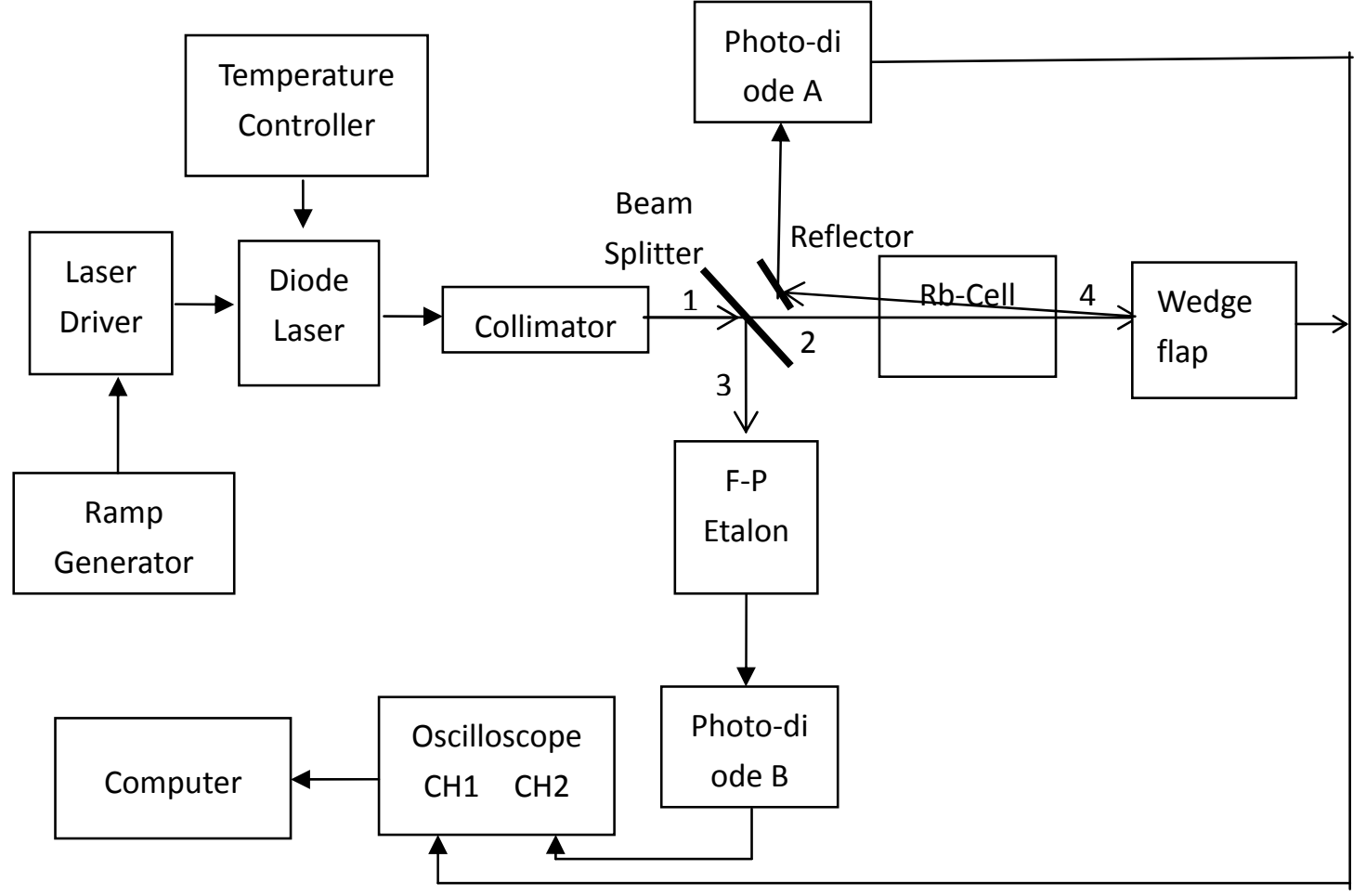

Fig. 4 Schematic diagram of the saturated absorption spectrometer. 


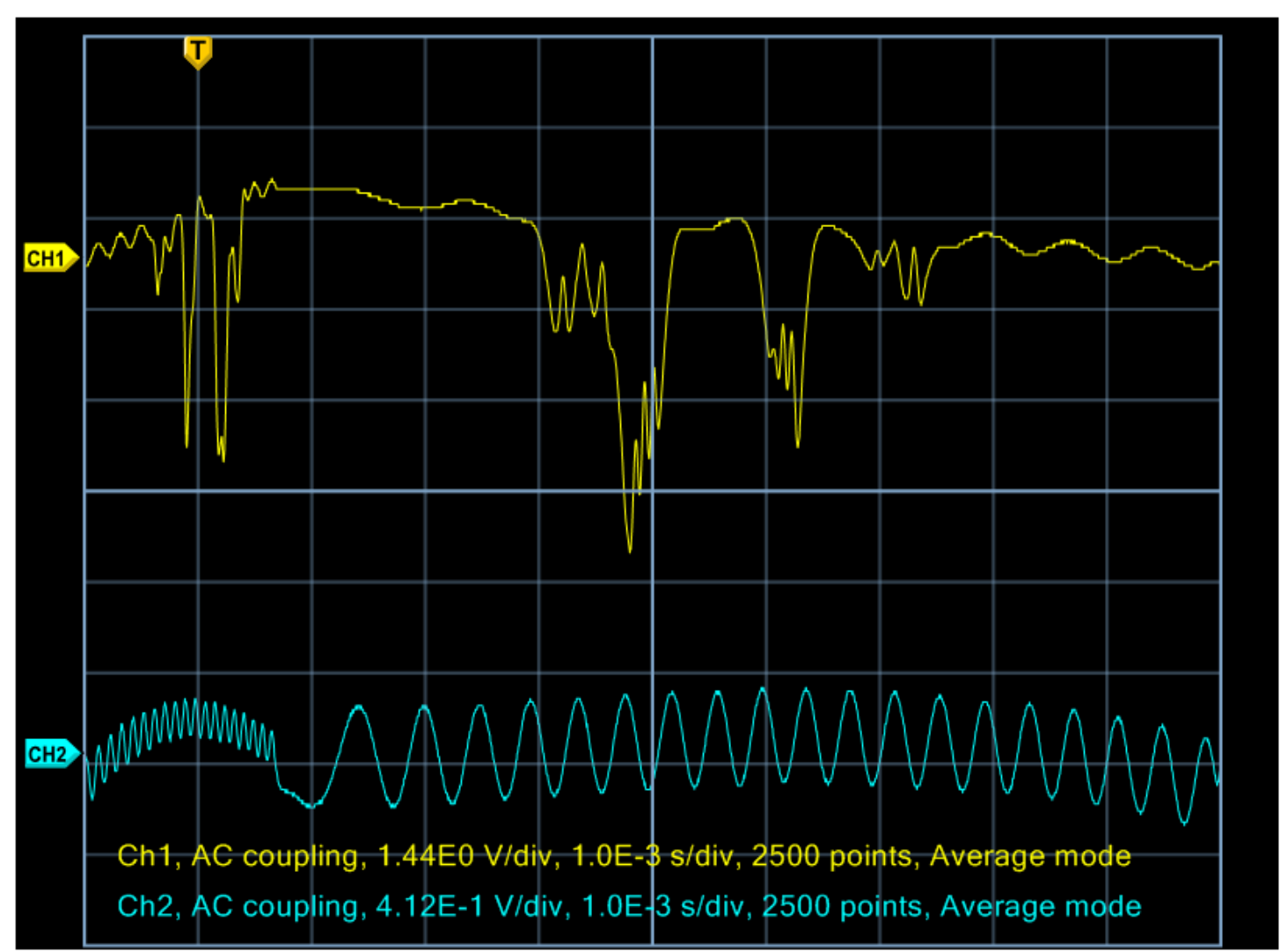

Fig. 5 Typical saturated absorption spectrum of Rubidium atom.

\section{Class demonstrative experiments}

Except the course experiments, we have prepared several interesting class experiments which demonstrate some simple spectroscopic technique.

Figure 6 shows the simple spectrometer connected with the camera. Students could see the spectrum of the light sources from the daily life like fluorescent lamps and LED. 


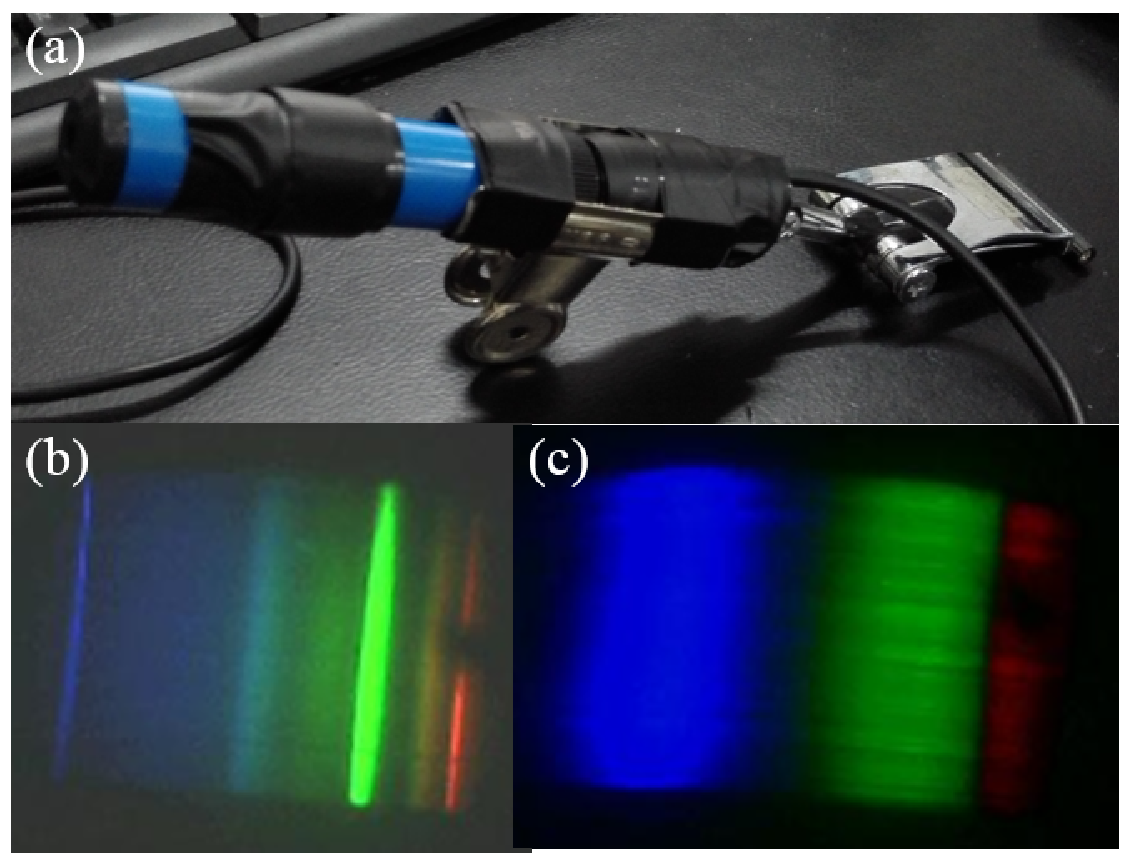

Fig. 6 (a) The picture of the simple equipment contains spectrometer and the camera. (b) and (c) are the spectroscopy of fluorescent lamps in the classroom and LED respectively.

Figure 7 shows how the prism splits the light out of the halogen lamp. It's a simple but fundamental demonstrative experiment which help students see how the spectrometer works visually.

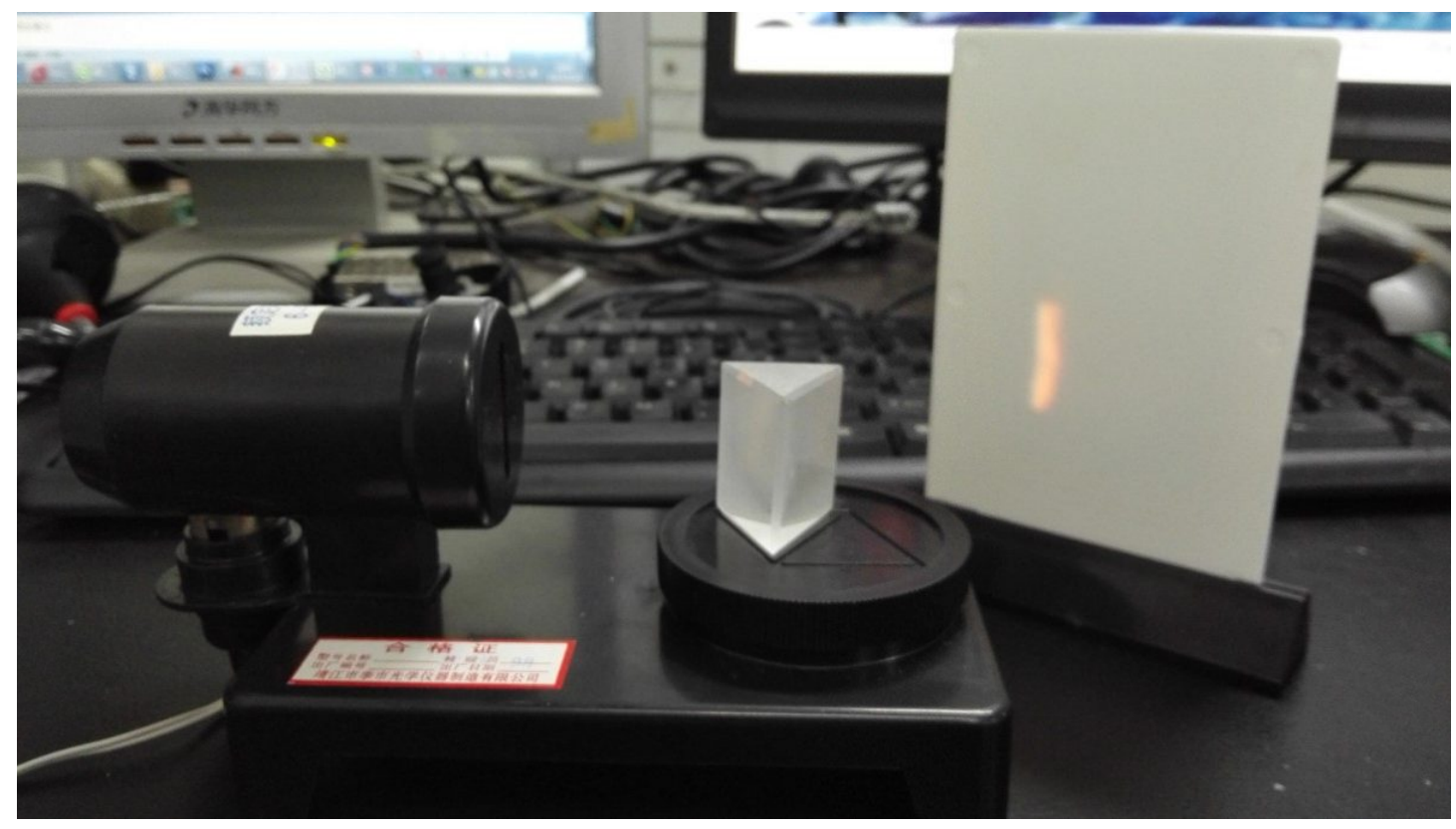

Fig. 7 The prism splits the light from the halogen lamp. 
What's more, we demonstrate the way to operate a portable and highly-integrated spectrometer (Figure 8), including setting up the experiment system, operating the software, showing how the spectrometer works, detecting spectrum of some goods and so on.

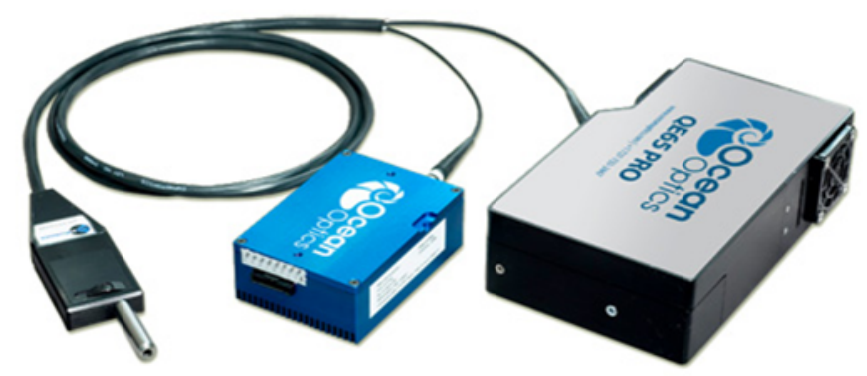

Fig. 8 Ocean Optics portable spectrometer.

To activate students' research interest, we organize them to play as atoms and imitate the molecular vibration. For example, in one game, two girls play as two oxygen atom meanwhile a boy plays as a carbon atom. Actually, the whole of them together is a molecule of carbon dioxide. They can do some types of movements to imitate the thermal motions and the different ways of vibrations such us stretching vibration, rotation and so on. What's more, students are encouraged to come up with some ideas of acting which could eliminate the Doppler-broadening.

\section{Conclusion}

In this report, we have outlined new approaches in teaching spectroscopy technique and applications: introducing history, experiments and frontier lectures simultaneously. Especially, the well-designed experiments and games, like diode laser based Doppler limited absorption spectroscopy, saturated absorption spectroscopy, and class demonstrative experiments, will stimulate students' interests a lot and these practice activities will bring them with more deep-going understanding of the spectroscopy.

\section{Reference}

1. A.V. Otieno, B.M. J. Muthoka. "Diode Laser Absorption Spectroscopy for Teaching Undergraduate Physics". IEEE.1247-1252 (1999).

2. Unknown, "Saturated Absorption Spectroscopy", Experiment SAS, University of Florida, Department of Physics PHY4803L, Advanced Physics Laboratory (2008). 